\title{
A HIGH FREQUENCY HIGH FLOW RATE PIEZOELECTRICALLY DRIVEN MEMS MICROPUMP
}

\author{
H.Q. Li, D.C. Roberts, J.L. Steyn, K.T. Turner, J. A. Carretero, O. Yaglioglu, \\ Y.-H. Su, L. Saggere, N.W. Hagood, S.M. Spearing, and M.A. Schmidt \\ Massachusetts Institute of Technology \\ Cambridge, MA 02139 \\ R. Mlcak \\ Boston Microsystems, Inc. \\ Woburn, MA 01801 \\ K.S. Breuer \\ Brown University \\ Providence, RI 02912
}

\section{ABSTRACT}

A piezoelectrically driven fluidic micropump was fabricated and tested. Microelectromechanical systems (MEMS) fabrication technology was used. Small cylindrical piezoelectric material elements were integrated with microfabricated silicon, silicon on insulator (SOI), and glass chips using eutectic bonding and anodic bonding processes. SOI wafers were used to form the thin membranes within the moving parts (a drive element and two passive valves). The design, microfabrication process, and assembly of the device are described in this paper. Fabrication issucs such as control of fillet radii at the fect of the Si membrancs for stress reduction and simultaneous eutectic and anodic bonding were discussed. A flow rate as high as $3000 \mu \mathrm{l} / \mathrm{min}$ was recorded. Experimental and simulation results of the dependences of the pump flow rate on the voltage and frequency applied on the piezoelectric material are shown and discussed.

\section{INTRODUCTION}

This paper presents the fabrication and testing of a high pressure, high flow rate piezoelectrically driven fluidic micropump with passive valves. In comparison to other piezoelectrically driven micropumps in the literature [1-4], this device incorporates the integration of bulk piezoelectric material with MEMS microfabrication techniques to allow for high structural driving frequencies in the tens of $\mathrm{kHz}$ range and implements novel methods of anodic and eutectic bonding to join silicon, glass, and piezoelectric components, enabling minimal device mass and therefore high power density. It requires a compact design that brings about challenging fabrication issues such as multi-layer wafer bonding, multiple lithography and deep reactive ion etch (DRIE) steps for the SOI wafers, use of nested oxide masks, and integration of small piezoelectric material elements. The need for high frequency and high flow rate calls for precisely micromachined Si membranes capable of large deflection as the moving components in the pump chamber and the passive valves of the micropump. SOI wafers are good choices for this purpose because they have a uniform thin Si layer, their buried oxide can be used as the etch stop during DRIE processes, and the fillet radii of the membranes that are critical to the strength of the membranes are easier to control in the presence of the buried oxide. Another advantage of SOI wafers is that separate electrodes can be formed in the SOI layer during microfabrication, with the buried oxide as the insulator. Eutectic bonding has been used to integrate the piezoelectric elements into the MEMS devices. For this micropump it is necessary to choose low melting point eutectic bonding alloys so that the eutectic bonding can be performed

sinultaneously with the anodic bonding of the $\mathrm{Si}$ and SOI layers to the glass layer that encloses the piezoelectric material for insulation. The successful fabrication of the micropump is a major step toward the realization of high power density transducer devices for micro-robotic actuation and heel-strike power generation applications $[5,6]$.

\section{MICROPUMP STRUCTURE}

The micropump has a multi-layer structure, schematically shown in Figure 1. It includes one fluid pump chamber and two identical passive check valves [7]. The volume change in the fluid chamber is achieved by the vertical motion of the double layer cylindrical piston-like drive element (layers 4 and 5) attached to annular $\mathrm{Si}$ membranes and driven by a ceramic piezoelectric cylinder (layer 3) $1 \mathrm{~mm}$ in diameter and $1 \mathrm{~mm}$ in height. When a voltage is applied to the piezoelectric cylinder, it pushes the drive element piston up, producing a high pressure in the fluid chamber that closes the inlet valve and opens the outlet valve so that fluid can flow out of the fluid pump chamber. Next, when the voltage is
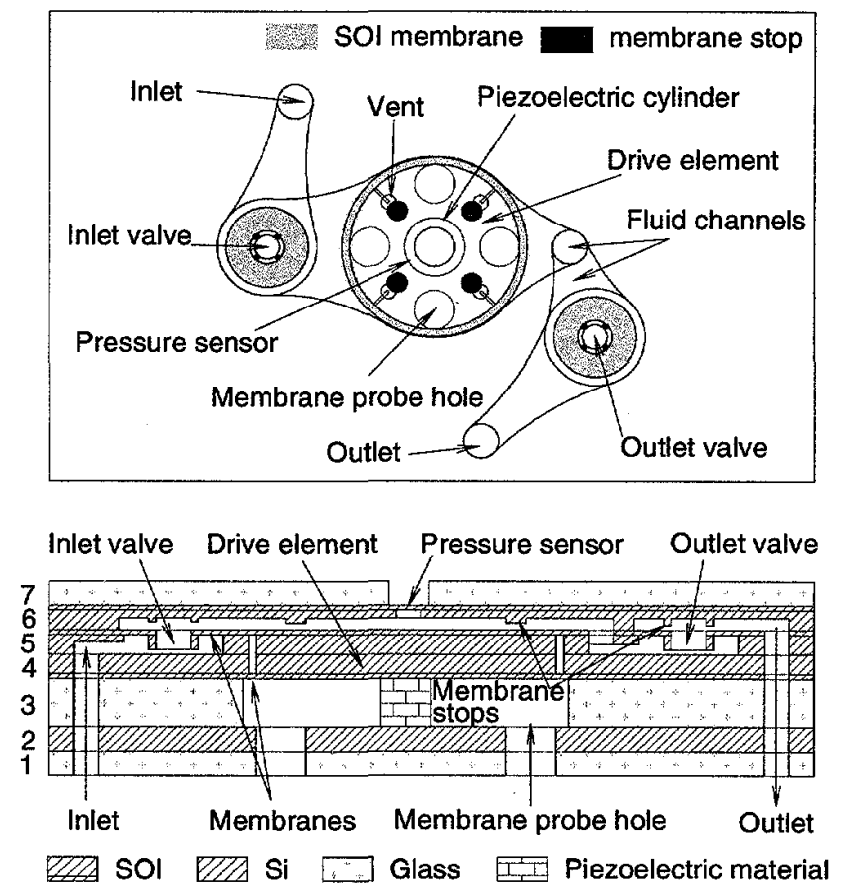

Figure1. Top view and cross-section view of the micropump. The cross-section view is from a zig-zag cut to show all the features. 
reduced the piezoelectric cylinder contracts and the drive element piston moves down, resulting in a low pressure in the fluid chamber that closes the outlet valve and opens the inlet valve so that fluid can flow into the chamber. The pump works by repeating these steps at high frequency.

The drive element piston is $3.6 \mathrm{~mm}$ in diameter and each of the two SOI layers 4 and 5 forming the piston is about $400 \mu \mathrm{m}$ thick. The annular drive element SOI membranes are $150 \mu \mathrm{m}$ wide and $10 \mu \mathrm{m}$ thick in the lower layer and $15 \mu \mathrm{m}$ thick in the upper layer which also includes the valves. SOI wafers (layers 4,5 , and 6) were chosen to construct the membranes in the device for two rcasons: 1) to control precisely the membrane thickness by using the buried oxide in the SOI as an etch stop in the DRIE process, and 2) to use the lower SOI (layer4) as an electrode to the piezoelectric cylinder and the buried oxide as an insulator between the piezoelectric cylinder and the remaining part of the micropump. The thickness of the SOI membranes in layer 5 was determined mainly by the design specifications of the passive valves that were subject to much larger deflections than the drive element piston membrane. Venting channels in layer 5 and holes in layer 4 (not shown in Fig. 1) were formed to release pressure in the cavity between the drive element membranes during the wafer diffusion bonding. The passive valves consist of a $0.5 \mathrm{~mm}$ center hole and a $15 \mu \mathrm{m}$ thick annular SOI membrane with an inner diameter of $0.70 \mathrm{~mm}$ and an outer diameter of $1.65 \mathrm{~mm}$. When not deflected the valves are only $0.5 \mu \mathrm{m}$ from layer 4 to minimize back leaking.

The fluid chamber above the drive element piston and fluid channels to and from the valves are located in layer 6 . Small cylindrical motion stops about $8 \mu \mathrm{m}$ from the drive element and about $18 \mu \mathrm{m}$ from the valves respectively were also formed in layer 6 to prevent the membranes from excessive deflections. A pressure sensor in the shape of a circular membrane $0.8 \mathrm{~mm}$ in diameter and $20 \mu \mathrm{m}$ thick was formed in the fluid chamber for onsite pressure measurement. A ceramic PZT-5H piezoelectric cylinder $1 \mathrm{~mm}$ in diameter and $1 \mathrm{~mm}$ in height was incorporated in the middle glass layer 3 as the active drive component of the micropump. The Si layer below the piezoelectric cylinder was used as the other electric contacts to the piezoelectric cylinder. $3 \mathrm{~mm}$ thick borosilicate glass was used in the top and bottom layers 1 and 7 for structural strengthening of this bench top device.

\section{MICROFABRICATION PROCESSES}

To minimize the size of the device, a compact design requiring a minimum number of layers, efficient use of the both surfaces in each layer, and therefore multiple lithography steps for the SOI wafers was implemented. As an example, Figure 2 shows the fabrication process flow for layer 5. The first step is the selection of the SOI wafer that has a $15 \mu \mathrm{m}$ thick SOI layer, a $0.4 \mu \mathrm{m}$ thick buried oxide, and a $380 \mu \mathrm{m}$ thick handle layer. Secondly, positive photoresist was coated on both sides of the wafer and the SOI side was patterned using standard lithography procedures. The photoresist on the handle side is to protect the handle surface from scratching in the lithography steps. This back surface protection method was used throughout the microfabrication processes. The wafer was then put in a DRIE machine until the SOI layer was etched through. This step formed the fluid through holes in the SOI layer. Next, the wafer was cleaned and coated with positive photoresist on both sides again. The handle side was patterned with photoresist and etched down about $0.5 \mu \mathrm{m}$ in a RIE etcher to form the clearance between the valves and their seating surfaces. In the fourth step the wafer was oxidized at $1100^{\circ} \mathrm{C}$ for 5 hours to form $2 \mu \mathrm{m}$ of thermal oxide that is to be used as a nested mask and back surface protection from DRIE etching later on. The thermal oxide was etched in step 5 to form the nested mask and then another lithography process was performed on handle side to define the membranes in the drive element and in the passive valves in step 6 . The wafer was etched first by DRIE about halfway though its thickness with photoresist as seen in step 6. Then the photoresist pattern was stripped and the nested thermal oxide mask was exposed for another DRIE step until it stopped at the buried oxide, as seen in step 7. The nested oxide mask was necessary to construct the $200 \mu \mathrm{m}$ deep venting channels in the drive element and the fluid channels to the valves. Finally, the thermal oxide was stripped using BOE wet etching.

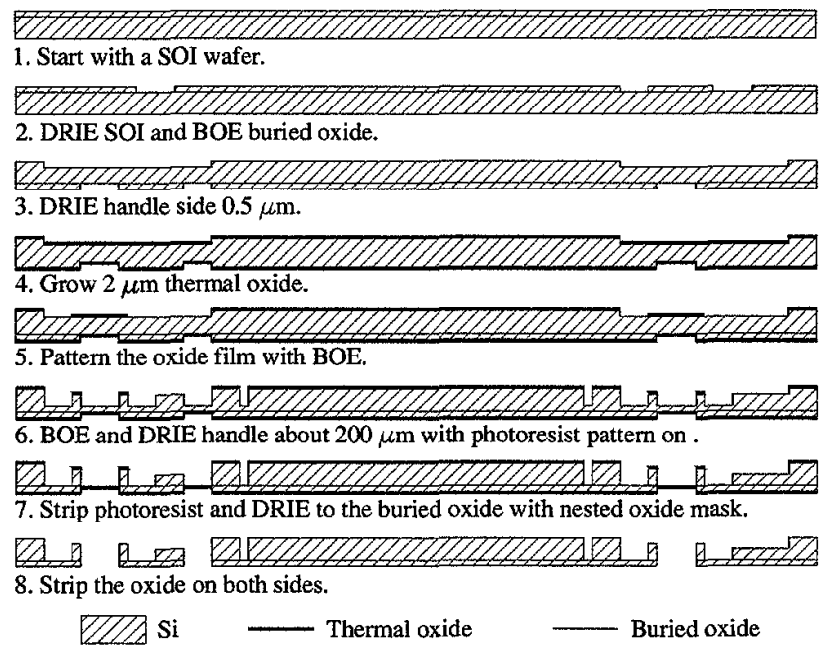

Figure 2. Fabrication flow of an SOI layer (layer 5 in Fig. 1) with the passive valves and half of the drive element piston.

Since all the membranes in this device have to operate under high stress and at high frequency, it is important to have a membrane structure with minimal stress concentration. A significant challenge in the micropump fabrication was controlling fillet radii in the range of $20-40 \mu \mathrm{m}$ for stress reduction at the feet of the membranes. In the DRIE machine $\mathrm{Si}$ is etched at a rate of about $2-4 \mu \mathrm{m} / \mathrm{min}$. depending on the feature size and exposed $\mathrm{Si}$ area. The etch front is usually a gradual curve in cross-section but varies depending on the etch conditions. An SOI membrane fillet radius is formed after the etch front reached the buried oxide and then the Si edges retreat horizontally to the side wall at a rate of about $70-100 \mu \mathrm{m} /$ minute as more oxide is exposed. There is a time window of only about 2-4 minutes to control the fillet radius in the desired range after about 2 hours etch of the handle side. Therefore careful monitoring in the final stage of the DRIE is critical. Figure 3 shows a $150 \mu \mathrm{m}$ wide drive element membrane with a gradual fillet about $25 \mu \mathrm{m}$ long horizontally.

\section{ASSEMBLY OF THE DEVICE}

After the final DRIE etch and cleaning, the three SOI wafers were aligned using an Electronic Visions system and fusion bonded at $1100^{\circ} \mathrm{C}$ in nitrogen for 1 hour. The bonded wafer stack was cut into $16.7 \mathrm{~mm}$ by $15.7 \mathrm{~mm}$ chips with a die-saw, cleaned in water, acetone, methanol, and isopropanol successively, and finally dried 
in air. The glass wafers were processed with diamond drills and die-saw cut into small chips. All the interfaces between $\mathrm{Si}$ and glass were anodically bonded at about $300^{\circ} \mathrm{C}$. The Si and the piezoelectric material interfaces were eutectically bonded.

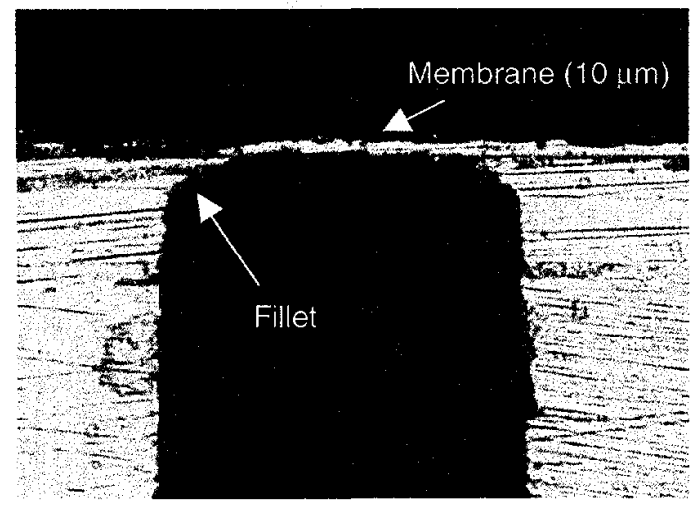

Figure 3. Optical cross-section picture of a drive element membrane, the membrane thickness is about $10 \mu \mathrm{m}$.

To prepare for the eutectic bonding, $500 \mathrm{~A}$ of Ti film and $5000 \AA$ of Pt film were deposited on the Si surfaces through a shadow mask by e-beam and $2 \mu \mathrm{m}$ of an Au-Sn (80-20\% wt.) alloy films were deposited on the piezoelectric surfaces by sputtering. The composition of the metal films were chosen for surface wetting (Ti) and low eutectic bonding temperature (Au-Sn) so that the eutectic bonding and the last anodic bonding can be done simultaneously as required by the pump design. The height of the piezoelectric cylinders were designed to be $2-3 \mu \mathrm{m}$ larger than the height of the middle glass piece (layer 3 in Fig. 1) so that the drive element piston is preloaded and when the chamber is expanded there is no pulling of the drive element piston by the piezoelectric cylinder that might delaminate the eutectic bonding. For this reason, an oxide mask that defines the piezoelectric seating was left on each Si chip below the piezoelectric (layer 2 in Fig. 1) for height compensation by individually trimming etch of the Si chips. Finally wires were soldered on the Ti/Pt electrode pads that were deposited at the same time as the eutectic bonding films were deposited. Figure 4 shows a picture of an assembled micropump.

The key components of this bench top device were built in an area of about $8.6 \mathrm{~mm}$ by $7.7 \mathrm{~mm}$, while the electric contacts and

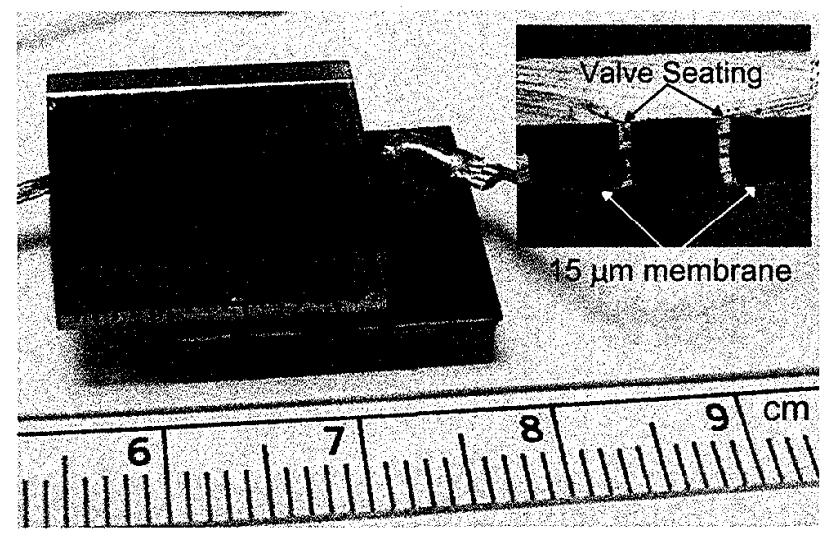

Figure 4. An assembled micropump with electric leads. Small graduations are in $\mathrm{mm}$. The inset is a cross-section picture of a passive valve with 15 m thick membrane. the supporting glass pieces take most space of this bench-top device. The inset of Fig. 4 is a cross-section photograph of a passive check valve with $15 \mu \mathrm{m}$ thick membrane.

\section{TEST RESULTS}

The assembled device was tested with an experimental setup that includes fluid reservoirs, valves, pressure sensors, and flow meters, as shown in Figure 5. The inset in Fig. 5 is an picture of the test jig with a micropump sealed in it by rubber $\mathrm{O}$-rings. The system was first evacuated and then filled with 1 centistoke silicone oil. The piezoelectric cylinders were driven by AC signals with peak to peak voltages in the range of $0-1600 \mathrm{~V}$, bias voltages in the range of $300-600 \mathrm{~V}$, and frequencies in the range of 1$12.5 \mathrm{kHz}$. The inlet pressure was maintained at $850 \mathrm{kPa}$ in all the tests. The highest flow rate recorded is $3000 \mu \mathrm{l} / \mathrm{min}$ at $1200 \mathrm{~V}$ peak to peak voltage, $600 \mathrm{~V}$ bias voltage, and $4.5 \mathrm{kHz}$, and zero differential pressure.

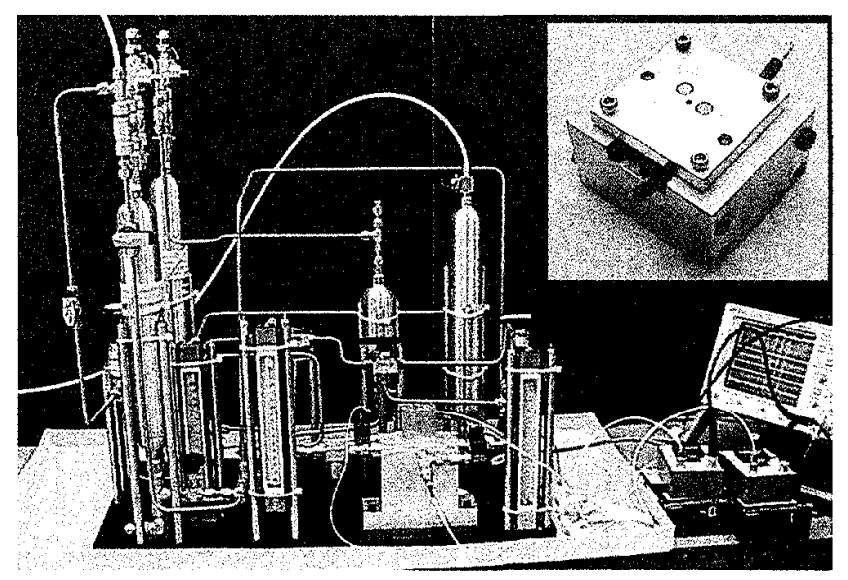

Figure 5. The experimental setup for micropump test. The inset is the package jig holding the micropump.

Figure 6 shows the experimental and quasi-static simulation results of the dependence of flow rate on peak to peak voltage. This test was done at a drive frequency of $3.5 \mathrm{kHz}$, a bias voltage of $600 \mathrm{~V}$, and zero differential pressure. The flow rate increases with the voltage monotonically but not linearly. In Simulation 1 a rigid chamber structure was assumed. It is believed that the flow rate of Simulation 1 is higher than the experimental results because the micropump has, as part of the fluid chamber, flexible SOI

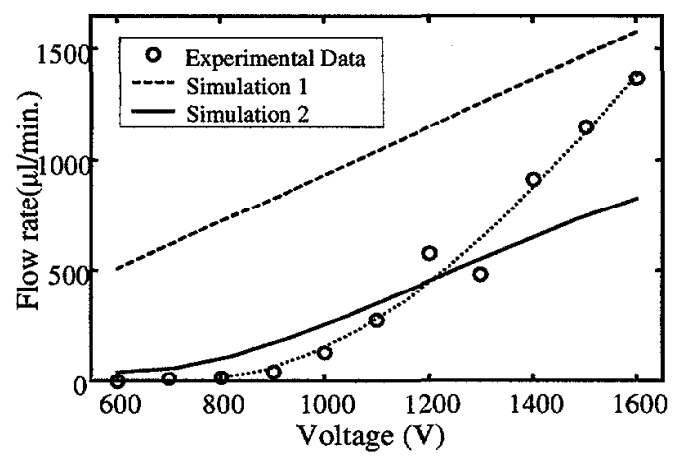

Figure 6. Flow rate vs. piezo peak to peak voltage at $3.5 \mathrm{kHz}$ drive frequency. The results of two quasi-static simulations with different chamber compliance are also shown. 
membranes attached to the drive element piston and the two passive check valves (see Fig. 1). An increased fluid chamber compliance was included in Simulation 2 and its results are closer to the experimental data as can be seen from Fig. 6. However, at high drive voltage the experimental flow rate increased with the drive voltage much faster than the results of Simulation 2. This is probably because the membranes deflect more and become stiffer as the drive voltage increases, resulting in a faster increase in flow rate, while in Simulation 2 this increase of membrane stiffness was not accounted for.

Figure 7 shows the relation between the inlet-outlet differential pressure and the flow rate. In this experiment the piezoelectric cylinder was driven at $1200 \mathrm{~V}$ peak to peak voltage, $600 \mathrm{~V}$ bias voltage, and $3.5 \mathrm{kHz}$.

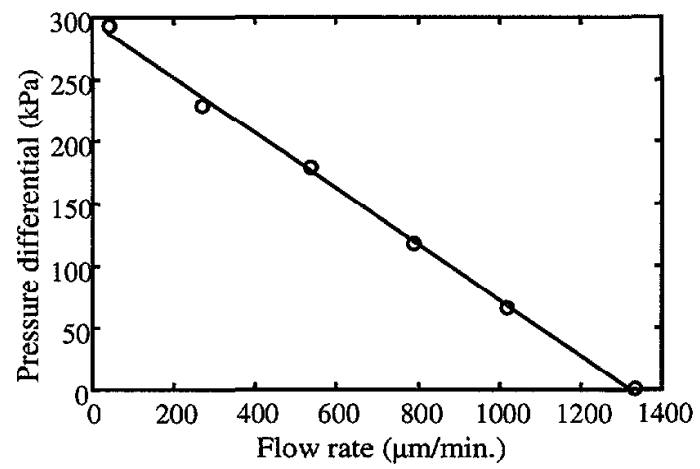

Figure 7 . Inlet to outlet differential pressure vs. flow rate.

The flow rate vs. piezoelectric cylinder drive frequency curve of the same device and a quasi-static simulation result are shown in Figure 8. Other experimental conditions of this test were: peak to peak voltage at $1200 \mathrm{~V}$, bias voltage at $300 \mathrm{~V}$, and zero differential pressure. Figure 8 clearly shows some form of resonance effect that is not present in the simulation. We found that changing the bias voltage to the piezoelectric material and changing the pressure difference between the inlet and outlet have an influence on the value of the flow rate but not on the resonance peak positions. The flow resonance could be due to the pressure wave effects at abrupt corners of the package of the micopump or due to the internal structure of the device. A systematic study of the micropumps is under way to better understand their behavior and to improve their performance.

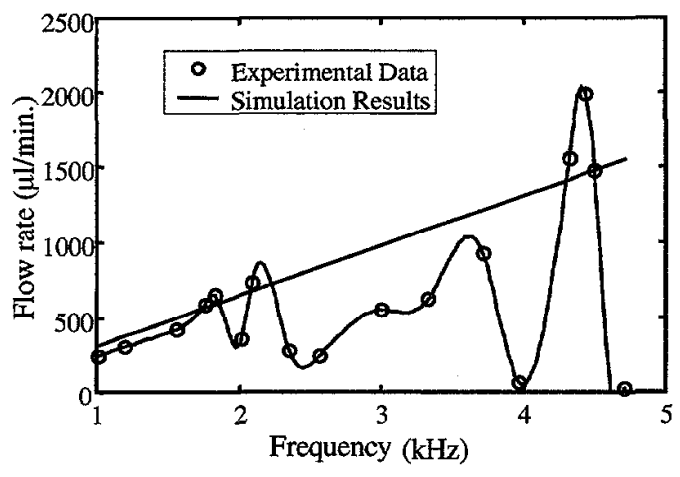

Figure 8. Flow rate vs. drive frequency of the micropump.

\section{CONCLUSIONS}

The work in this paper is part of a larger effort to develop high power density transducer devices [5] for micro-robotic actuation and human heel-strike power generation applications. The successful fabrication of the micropump has validated the MEMS process design and bonding techniques to be used for these devices. Since device power is generally proportional to flow rate, higher flow rate micropumps with larger drive elements and multiple piezoelectric cylinders are currently being developed. A further increase in flow rate can be realized by using newly developed single crystal piezoelectric materials [8]. A performance improvement of 10 times is expected in micropumps that combine larger drive elements and single crystal piezoelectric materials. In order to examine the effect of resonance on the performance of the micropump, the internal device structure and external test set-up are under investigation. In addition, it may be possible to use this resonance behavior to maximize the flow rate by tuning the device to a desired resonance frequency.

\section{ACKNOWLEDGEMENT}

We would like to thank the staff members of the Microsystems Technology Laboratories at MIT, especially Kurt Broderick, for their help in the microfabrication of the micropumps. We also thank Dr. Arturo Ayon of MIT for his help with the diffusion bonding of the SOI wafers. This work is funded by DARPA.

\section{REFERENCES}

1. S. Shoji and M. Esashi, "Microflow Devices and Systems", Journal of Micromechanics and Microengineering, 4, 157, (1994).

2. M. Koch, A.G.R. Evans, and A. Brunnschweiler, "The Dynamic Micropump Driven with a Screen Printed PZT Actuator", Journal of Micromechanics and Microengineering, 8, 119, (1998).

3. M. Esashi, S. Shoji, and A Nakano, "Normally Closed Microvalve and Micropmp Fabricated on a Silicon Wafer", Sensors and Actuators, vol. 20, pp 163-169, 1989.

4. D. Maillefer, H. Van Lintel, G. Rey-Mermet, R. Hirschi, "A High Performance $\mathrm{Si}$ Micropump for an Implantable Drug Delivery System", Techinical Digest, IEEE International MEMS'99 Conference, pp. 541-6, (1999).

5. Nesbitt W. Hagood IV, et al. "Micro-hydraulic Transducer Technology for Actuation and Power Generation", submitted to SPIE $7^{\text {th }}$ International Symposium on Smart Structures and Materials, Newport Beach, CA, March 5-9, 2000.

6. D.C. Roberts et al. "Design of Piezoelectrically-driven Hydraulic Amplication for High Pressure, High Frequency Applications", submitted to SPIE $7^{\text {th }}$ International Symposium on Smart structures and Materials, Newport Beach, CA, March 5-9, 2000.

7. H.T.V. Van Lintel, F.C.M. Van De Poll, and S. Bouwstra, "A Piezoelectric Micropump Based on Micromachining of Silicon", Sensors and Actuators 20, 163, (1989).

8. Seung-Erg Park and Thomas R. Shrout, "Characteristics of Relaxor-Based Piezoelectric Single Crystals for Ultrasonic Transducers", IEEE Trans. Ultrasonics, Ferroelectrics, and Frequency Control, 44, 1140 (1997). 